\title{
Latent Profile Analysis in R: A tutorial and comparison to Mplus
}

\author{
Klaas J. Wardenaar \\ University Medical Center Groningen (UMCG) \\ k.j.wardenaar@umcg.nl
}

April 9, 2021

Version 1.1

\section{Summary}

Latent profile analysis (LPA) can be used to identify data-driven classes of individuals based on scoring patterns across continuous input variables. LPA can be conducted using commercially available software packages like Mplus, Latent Gold, and SAS, but it is also possible to use freely available R-packages. This tutorial aims to (1) help applied researchers to conduct an LPA in R and (2) to show how results obtained in $\mathrm{R}$ compare to those obtained in Mplus.

\section{Background}

Latent profile Analysis (LPA) is a type of latent variable model that can be use to identify latent classes or mixtures in a dataset, based on a set of continuous input variables (Gibson, 1959; Oberski, 2016). LPA is closely related to the widely used technique of Latent Class Analysis, which is used to estimate latent classes based on discrete input variables (NylundGibson \& Choi, 2018). In medical and psychological science, LPA can be useful when considerable between-subject heterogeneity exists in scores on a range of variables and when this variation cannot be explained by known, manifest variables (e.g., Wolfe 1970; Sterba 2013). Here, LPA can help to identify or approximate possibly meaningful sub-grouping of subjects that may help to better understand sample heterogeneity (Sterba 2013).

Generally, LPA works under the assumption that sample (residual) variance can be reduced by assuming a categorical latent variable that effectively subdivides the sample into $>=2$ subgroups that are more homogeneous in terms of their patterns of variable means and (co)variances. In case an LPA model is found to fit a dataset well, it is often the case that subjects in each class resemble each other relatively well in terms of their scores on the input variables. Depending on the model configuration, the identified classes can show different class-specific patterns of means and class-specific or class-varying variances. 
Many different LPA model configurations are possible, each with different sets of parameters that are either freely estimated in each class specifically or constrained to be equal across classes in the resulting model (e.g., Celeux \& Govaert, 1995). Model configurations with many class-specific parameters can be very flexible and, as such, may fit the data well. A downside is that these models are more complex (much more parameters to be estimated). Using criteria, such as the Bayesian Information criterion (BIC) helps to find a model that strikes a good balance between model-fit and model-complexity. When doing an LPA, most applied researchers will usually be most interested in the differences and/or overlap between the classes' specific patterns of parameter estimates. These can be used to characterize the classes and, possibly, provide clues about underlying mechanisms (Sterba 2013).

\section{This tutorial}

LPA is less widely used than other latent variable models and, possibly due to that, has long been only available in specialized software packages such as Mplus. Luckily, ongoing developments in many different scientific fields (e.g., ecology, econometrics) have yielded a number of packages that also allow users to conduct LPA in the open-source R-platform. However, the use of $\mathrm{R}$ does require experience and documentation of packages can be rather limited or technical, making it less easily accessible for applied researchers. Therefore, this tutorial is aimed to help applied researchers to get going with LPA in R, illustrating the use of several packages and, for reference, providing a comparison of the results obtained in $\mathrm{R}$ with results obtained with Mplus.

\section{Data}

All examples in this tutorial will be using a simulated dataset (see Appendix for code). The simulated data consist of 300 cases, each with responses on 10 continuous variables. The data are simulated to consist of 3 classes, each with different mean scores across the 10 variables and each with a different variable (co)variance matrix. The following figure shows the simulated data, with the different colors indicating the different classes. 


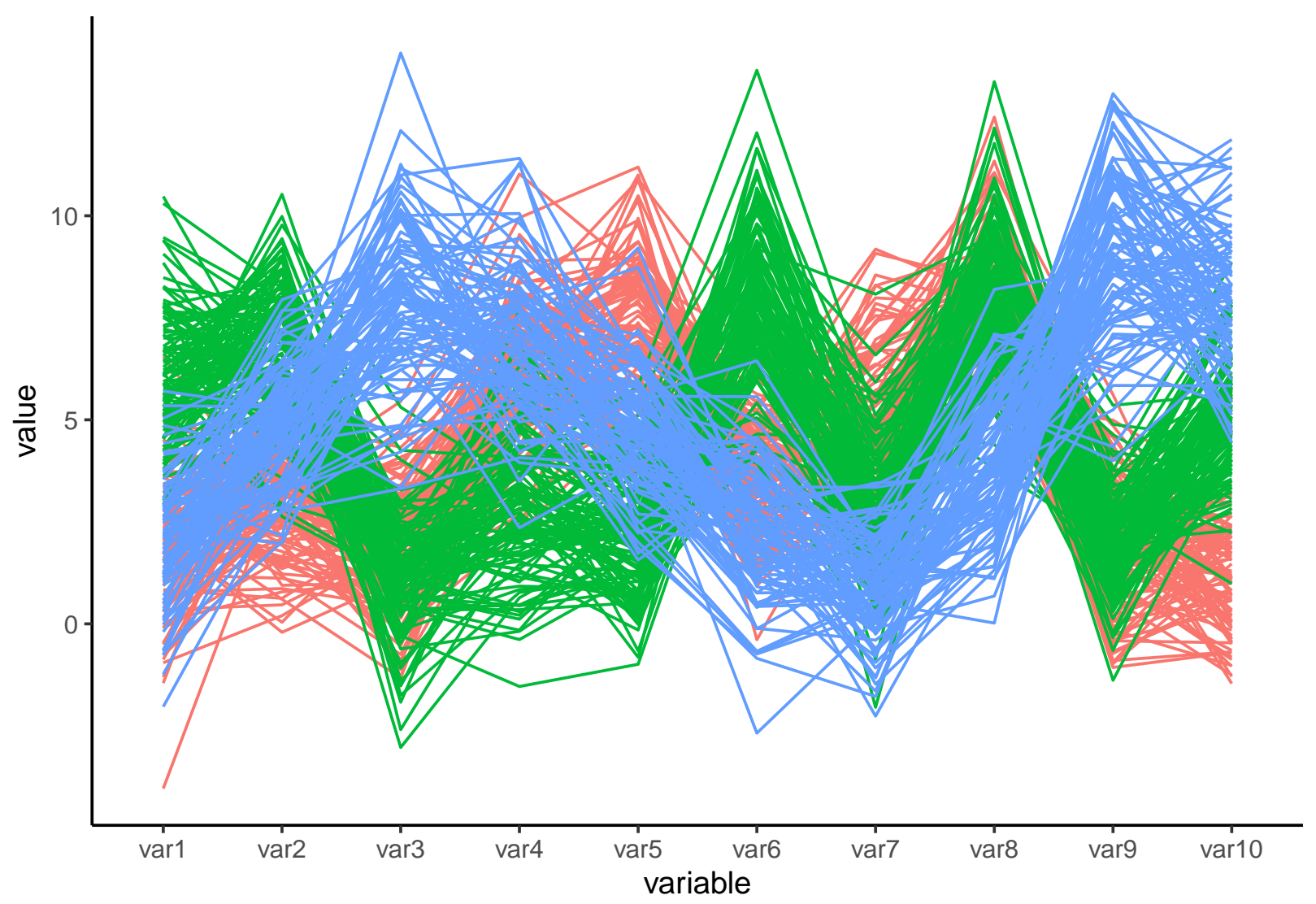

Note that the data were simulated to have a latent structure that is quite obvious. Even if the classes' lines in the plot had the same color, some clustering would still be observable. In real life research settings, data with such obvious patterns are never - or seldom - encountered.

\section{Packages}

\subsection{R-packages}

LPA models can be fit in $R$ (version 4.0.3; R-core team, 2020), running in Rstudio (version 1.3.1093 used here). There are many R-packages that offer some form of latent class/mixture analytic functionality. However, the majority of packages is focused on analyses with discrete indicator variables (e.g., 'poLCA', Linzer \& Lewis, 2011; 'e1071::lca', Meyer et al., 2019) or require a lot of coding to define the required models (e.g., openMX, Neale et al., 2016). For the current tutorial, two packages were selected that work with continuous indicator variables and that require limited user coding. 'mclust' package (Scrucca et al., 2016) is the first package that will be illustrated. This package is a specialist tool and allows for a wide variety of model configurations to be estimated; as such it offers much more functionality than most researchers will likely need. Therefore, the current tutorial focuses on a limited range of relatively simple LPA model configurations that are commonly encountered in the literature. Conveniently, Rosenberger et al., (2018) recently developed the package 'tidyLPA' which can be used as a relatively easy to use front-end for estimating common LPA models with 'mclust', basically streamlining some of the in- and output functionality of ' $m c l u s t$ '. 
Although 'tidyLPA' is easy to use, this comes at the expense of restricting modeling options to a few, oft-used options. For completeness, both the 'mclust' and 'tidyLPA' approach will be illustrated.

\subsection{Mplus}

Because it is one of the most widely used commercial software packages for latent variable modeling, Mplus (version 5, Muthén \& Muthén, 2010) is used to fit some of the same LPA models as are estimated using the two R-packages. The results obtained in Mplus are compared to the results obtained with $\mathrm{R}$ and the extent of overlap and/or differences between the software packages is evaluated.

\section{Model configurations}

LPA models can be configured in many different ways (see Scrucca et al., 2016; Banfield \& Raftery, 1993; Celeux \& Govaert, 1995; Pastor et al., 2007). Here, four variants of increasing complexity will be covered. In all models, cluster-specific means are estimated for each of the k classes: each class has its own associated pattern of mean scores on the indicator variables (e.g., var1-var10). The different model versions vary in terms of how the class-specific (co)variance matrices of the indicator variables are constrained or allowed to vary within and between classes. See the following table for an overview of the three models:

\begin{tabular}{l|l|l|l|l}
\hline \multirow{2}{*}{ Model variant } & \multicolumn{2}{|c|}{ Variances } & \multicolumn{2}{c}{ Covariances } \\
\cline { 2 - 5 } & vary within class? & vary beteen class? & vary within class? & vary beteen class? \\
\hline EEI & yes & no & no; fixed to 0 & no; fixed to 0 \\
\hline EEE & yes & no & yes & no \\
\hline VVI & yes & yes & no; fixed to 0 & no; fixed to 0 \\
\hline VVV & yes & yes & yes & yes \\
\hline
\end{tabular}

In the first, most parsimonious LPA model variant, the indicator variables are set to have zero covariances within and across classes. Indicator-variable variances are allowed to vary within classes but are constrained to be equal between classes. Due to the latter, only one set of variances needs to be estimated, resulting in a parsimonious model. In 'mclust', which uses Gaussian Mixture Modeling vernacular, this variant is also referred to as the EEI (equal volume, equal shape [and undefined orientation]) model (Scrucca et al., 2016).

The second model resembles the first model, but here the complete variable (co)variance matrix is estimated: i.e. both the indicator variances and covariances are estimated. As in the first model, the resulting (co)variance matrix is constrained to be equal across classes. In 'mclust' jargon, this type of model is called an EEE (equal volume, equal shape, and equal orientation) model.

The third model variant allows for more variation across classes. All within- and between class variable covariances are set to zero, but variances are now allowed to vary within and between classes. As a result, the number of variance parameters to be estimated increases with each class that is added to the model. In 'mclust', this type of model is called a VVI (varying volume, varying shape [and undefined orientation]) model. 
The fourth, most complex model allows for most variation in (co)variances across classes: both the variances and covariances are allowed to vary within and between classes, resulting in estimation of class specificcovariance matrices. This type of model is called a 'VVV' (varying volume, varying shape, varying orientation) model .

Of course it is possible to design many other LPA models that, for instance, use partially constrained (co)variance matrices across classes. The 'mclust' package alone covers an additional 10 different model variants. Mplus, which takes a different approach to LPA than 'mclust', also allows for additional and highly customized model variations. These are not covered here, but will be relatively easy to implement for users once they are familiar with the four basic variants mentioned above.

\section{Tutorial}

\section{1 'mclust'}

To run LPA using the 'mclust' package, the Mclust() function can be used. This function requires data as its minimum input. In addition, the number of clusters to fit $(\mathrm{G})$ and model variants to fit and select from (modelNames) can be entered (defaults are: G=1-9 and all fourteen model variants). In many cases, we may want to consider a more limited range of model variants to with different numbers of classes. For multivariate data, a total of fourteen options are available (see help ("mclustModelNames") for an overview). Each model variant is estimated for each value of the specified range of G using Expectation Maximization (EM), using the Bayesian Information Criterion (BIC) to compare different model variants and select the optimal one.

Conducting a latent variable model or mixture analysis with Mplus or comparable software usually entails fitting models with different numbers of classes (that are otherwise configured similarly) and to compare the fit between these models to select the best model. The default approach taken in the 'mclust' package is slightly different in that the main modeling command Mclust () fits multiple models for all possible combinations of the specified number of classes and model configurations. The eventual output of 'mclust' is the BIC-selected best model variant with the optimal combination of number of classes and model configuration.

An advantage of the 'mclust' approach is that it is efficient and flexible: given a number of classes, the best model configuration is selected straight away and we do not have to run all model combinations one by one. This makes the approach especially suitable for exploratory analyses. However, like stated above, this approach is somewhat less usual in some fields, where estimation of LPA is approached in a more confirmatory fashion. In addition, it does take some control away from the researcher, who may want to primarily focus on selecting the number of classes, given a particular, theory-based model configuration that is kept constant throughout analyses, which is often the chosen strategy in software like Mplus and made easily available through the 'tidyLPA' package.

Here, both approaches are shown: (1) the 'mclust' aproach and (2) the 'tidyLPA' approach. 


\subsubsection{The 'mclust' approach}

In this approach, we run consecutive models with increasing numbers of classes $(\mathrm{G}=1$ to $\mathrm{G}=9$ ), for each model we let the package fit the four above described model variants ("EEI", "EEE", "VVI" and "VVV") and select the best-fitting one. We start by loading the package (library (mclust)) and creating an object mnames that contains the names of the four models we want to be fitted. Next, we use the Mclust() function to fit the models:

library(mclust)

\#\# Package 'mclust' version 5.4.7

\#\# Type 'citation("mclust")' for citing this $R$ package in publications.

mnames <- c("EEI", "EEE", "VVI", "VVV")

\# Fit 1-5 class model

mod_g1_9 <- Mclust (dat1 $[, 1: 10]$, modelNames = mnames)

We can first look at the optimal number of classes and the optimal model variant that were selected, using the following code:

\# Opimal number of classes

mod_g1_9\$G

\#\# [1] 3

\# Optimal model variant

mod_g1_9\$modelName

\#\# [1] "EEI"

This shows us that a 3-class EEI model was selected as the best model based on the BIC. We can get a better perspective of this model's performance if we compare it to the other fitted models. We can do this by taking a closer look at the other models' BIC values:

mod_g1_9\$BIC

\#\# Bayesian Information Criterion (BIC):

\#\# EEI $\quad$ EEE VVI VVV

\#\# $1-14661.61-12986.57-14661.61-12986.57$

\#\# $2-13275.65-12611.60-13095.82-12678.12$

\#\# $3-12142.11-12290.68-12163.08-12731.18$

\#\# $4-12178.44-12330.04-12241.21-12970.57$

\#\# 5 -12223.14 $-12379.96-12320.47-13231.35$

\#\# $6-12264.04-12423.72-12406.59-13522.40$

\#\# 7 -12269.39 $-12451.25-12497.03-13748.91$

\#\# 8 -12298.30 $-12483.35-12568.62-13980.82$

$\begin{array}{llllll}\text { \#\# } 9 & -12347.11 & -12529.40 & -12645.50 & -14269.34\end{array}$

\#\# 


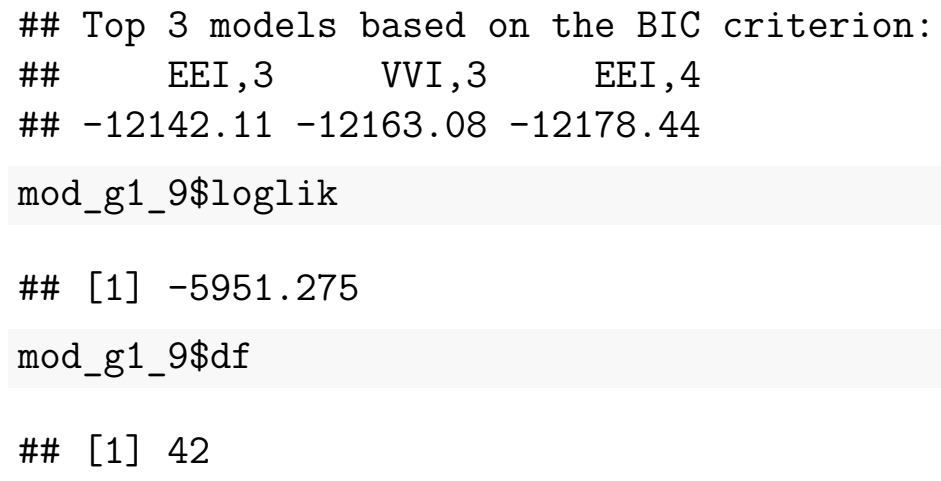

Here, we can see the BICs for all fitted models (in this case 1-9 classes and 4 model variants: 36 models in total). We can see that the closest contenders were a 3-class model with a VVI configuration (with variances allowed to vary both within and between classes) and a 4-class EEI model.

Note that the BIC values are negative, and that values that are more negative are considered to fit poorer than values closer to 0 . This may strike some users as odd, given that in many modeling applications, we are used to consider lower BIC values to indicate better fit. However, the 'mclust' approach of maximizing the BIC is correct for the used modeling approach (see e.g., Fraley \& Raftery, 2003; Banfield \& Raftery, 1993). For theoretical reasons, the BIC is calculated in'mclust' as:

$$
B I C=2(\text { Loglikelihood })-d f(\log (n))
$$

where $d f$ is the number of parameters (degrees of freedom) and $n$ is the sample size. We can see, that here, higher loglikelihood values lead to a higher BIC. For the best fitting model in our example, the loglikelihood is -5951.275 , the number of parameters is 42 and the sample size is 300 . If we plug these into the formula, we get the following BIC:

$$
B I C=2(-5951.275)-42(\log (300))=-12142.11
$$

As expected, the obtained value indeed corresponds to the BIC we got in the model output (see above). Other software packages, such as Mplus and 'tidyLPA' (see below) use a slightly different formula to calculate the BIC, where higher loglikelihood values lead to lower BIC values:

$$
B I C=d f(\log (n))-2(\text { Loglikelihood })
$$

When we plug the best-model values into this formula, we get:

$$
B I C=42(\log (300))-2(-5951.275)=12142.11
$$

We can see that the absolute BIC value is the same, irrespective of the used formula, with only the sign differing between the two approaches. 
Now, let us continue by taking a closer look at the class sizes and the mean values and variances of the ten variables for the three identified classes. Because the best model has and EEI configuration, printing out a single variance matrix from one class will suffice, because the variances are the same across classes (this is different in cases where the optimal model allows for between-class differences in (co)variances:

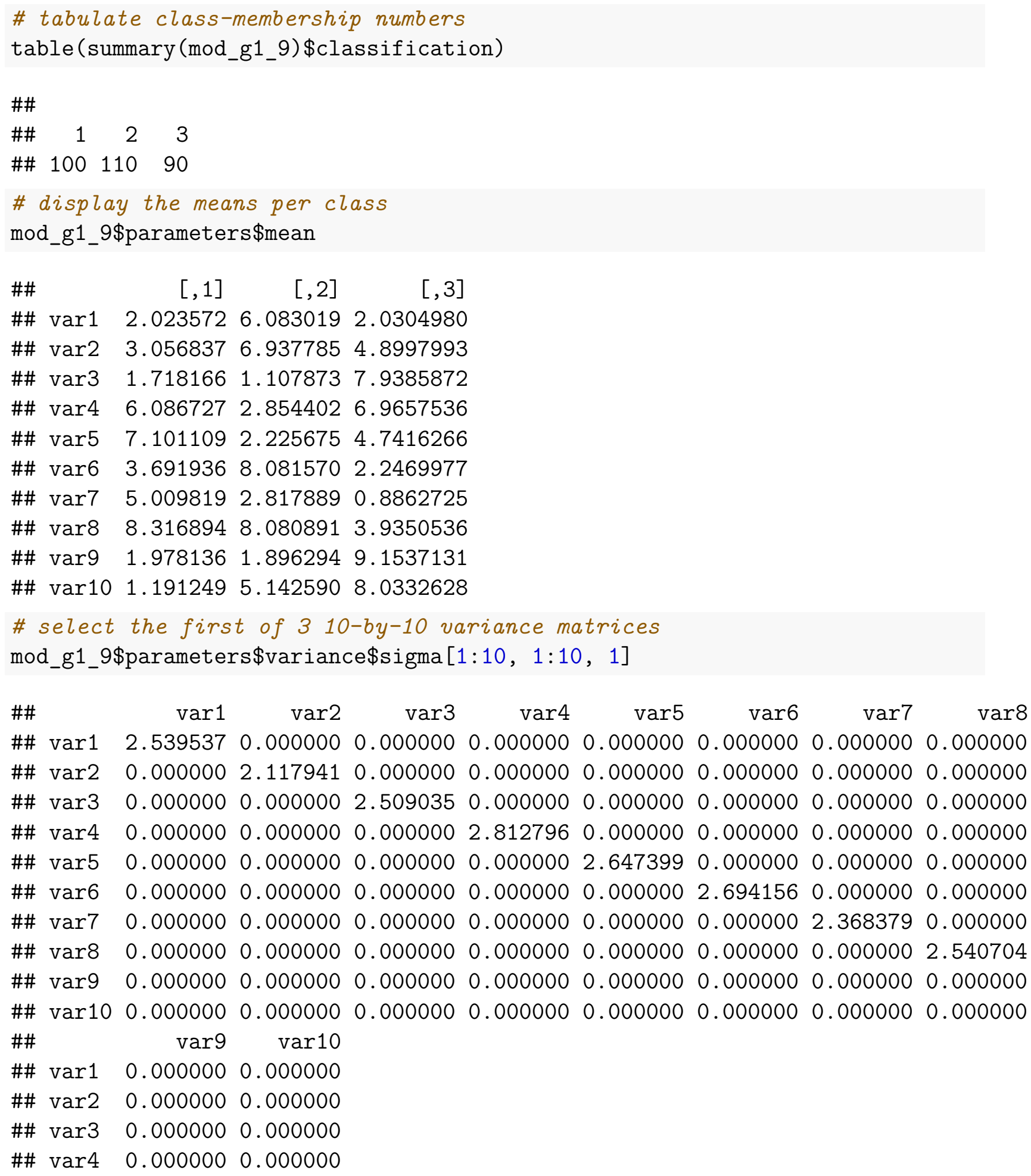




$\begin{array}{llll}\text { \#\# } & \operatorname{var} 5 & 0.000000 & 0.000000 \\ \text { \#\# } & \operatorname{var} 6 & 0.000000 & 0.000000 \\ \text { \#\# } \operatorname{var} 7 & 0.000000 & 0.000000 \\ \text { \#\# var8 } & 0.000000 & 0.000000 \\ \text { \#\# } \operatorname{var} 9 & 2.509146 & 0.000000 \\ \text { \#\# } & \operatorname{var} 10 & 0.000000 & 2.204457\end{array}$

From this output, we can see that the three classes have class-sizes of 100, 110 and 90, respectively. These sizes along with the patterns of class-specific mean variable scores correspond with those that served as the input for the simulation. As specified, the variances vary slightly across variables.

The class allocation in LPA is probabilistic in nature: each subject in the data is assigned a probability for each of the estimated classes, based on their pattern of scores on the input variables. These probabilities can be inspected in the $z$-matrix (here: mod_g1_9\$z). Subjects can be allocated to one of the classes based on their highest class-probability. These posterior allocations can be found in the classification matrix (here: mod_g1_9\$classification). These class-allocations were tabulated above to evaluate class sizes.

Note that class-allocation in this way only yields useful classifications if the patterns of class-probabilities allow for allocation of each subject to a single class with sufficient certainty. In case of too much uncertainty, using the classification is not advised. We can inspect the uncertainty of allocation for all subjects (here: mod_g1_9\$uncertainty). This gives us a list of uncertainties for all subjects. Next, we can evaluate the extent of uncertainty by looking at the maximum uncertainty or, for instance, the averaged uncertainty across subjects:

$\max ($ mod_g1_9 $\$$ uncertainty)

\#\# [1] 0.003852604

mean(mod_g1_9\$uncertainty)

\#\# [1] 2.607299e-05

Here, the uncertainty is atypically low because of the used idealized data. In many cases, uncertainty is higher and it may be of interest to investigate it further, for instance, by looking at (e.g., plotting or tabulating) the uncertainty per class:

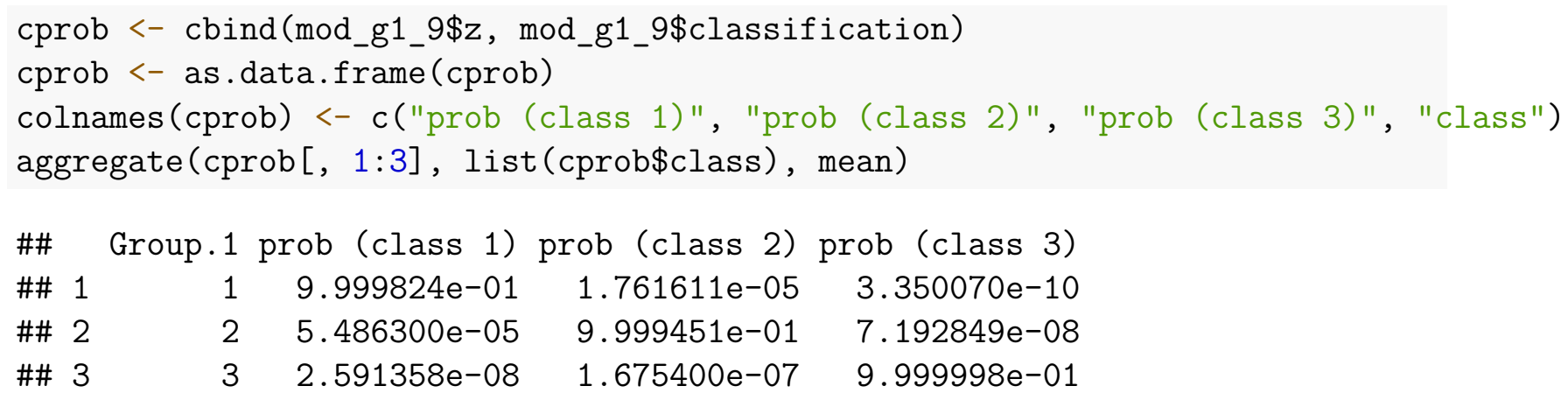

Here we can see that for each class, the mean probability of membership of that class was 
very high and probabilities for the other classes were very low. Again, these values reflect the idealized data; in many cases, more differentiated class-probability patterns may be observed.

\subsection{2 'tidyLPA'}

When running ' $m$ clust' models from the 'tidyLPA' package, the estimations are approached a little differently. By default, the number of models that can be estimated is restricted to six relatively common variants (see help(tidyLPA: :estimate_profiles) for details). Of these models, four can be estimated with 'mclust'. These models differ in terms of how the variances and covariances are allowed to vary or constrained to be equal across classes and whether covariances are or are not fixed to zero within classes. These models have each been allocated a number (1-6) in 'tidyLPA'. See the table below how these numbers correspond to the above mentioned model configurations.

\begin{tabular}{l|l}
\hline Model variant & model number \\
\hline EEI & 1 \\
\hline EEE & 3 \\
\hline VVI & 2 \\
\hline VVV & 6 \\
\hline
\end{tabular}

Models four and five are different and can only be fit when $\mathrm{R}$ is interfacing with Mplus using the 'MplusAutomation' package. This is outside the scope of this tutorial.

To fit an LPA model in 'tidyLPA', we use the estimate_profiles() function. Here, we need to enter the data.frame to be used ( $\mathrm{df}$ ) and the number of classes to estimate (n_profiles). To determine what kind of model configuration to estimate, the authors have provided two different ways in the estimate_profiles() command. In the first approach, we simply tell what model number (see Table 2) we want to estimate using the models= argument For instance, if we want to estimate LPA models with 1 to 9 classes, with an EEI configuration, we can use:

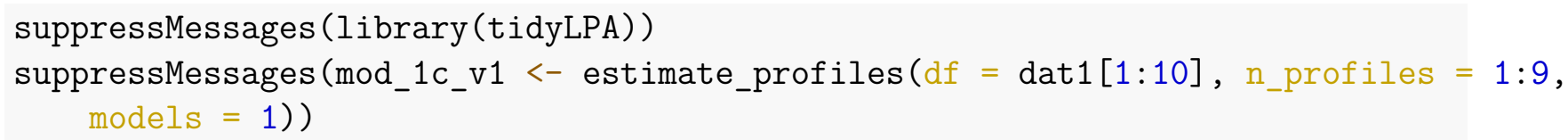

The package issues a standard message by default notifying us that we use the models argument and that the variances and covariances arguments are ignored. Here, we suppress the message. The output shows us the model-fit information for the 1 to 9-class EEI models:

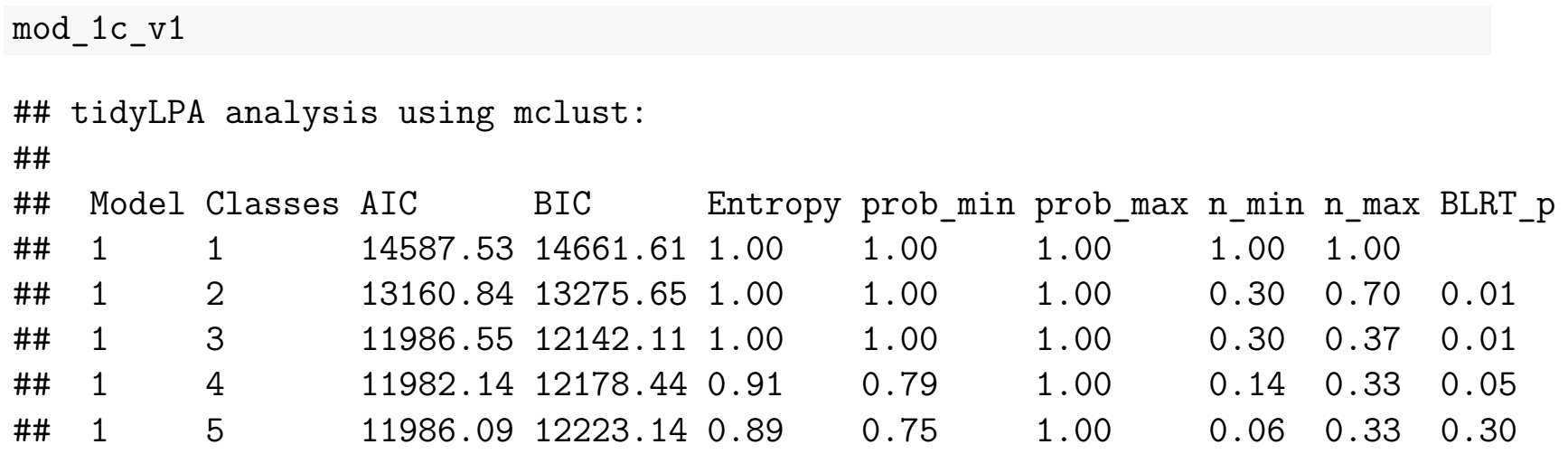




$\begin{array}{lllllllllll}\# \# & 1 & 6 & 11986.25 & 12264.04 & 0.84 & 0.78 & 1.00 & 0.08 & 0.30 & 0.16 \\ \# \# & 1 & 7 & 11950.86 & 12269.39 & 0.81 & 0.77 & 0.91 & 0.08 & 0.17 & 0.01 \\ \# \# & 1 & 8 & 11939.04 & 12298.30 & 0.82 & 0.79 & 0.92 & 0.08 & 0.17 & 0.02 \\ \# \# & 1 & 9 & 11947.10 & 12347.11 & 0.82 & 0.76 & 0.92 & 0.06 & 0.16 & 0.57\end{array}$

Here, we can see that the BIC takes on different values compared to 'mclust', with lower rather than higher values indicating best fit (see explanation above). Instead of drawing the fit indices (e.g., BIC, AIC) directly from the 'mclust' package, 'tidyLPA' only draws the 'raw' loglikelihood values and posterior probabilities from the 'mclust' output and (re)calculates the BIC, AIC, entropy etc. to mirror as well as possible those provided in Mplus. In addition, p-values of the the bootstrapped likelihood ratio test (BLRT) are given by default. These $\mathrm{p}$-values indicate for each $\mathrm{k}$-class model whether adding the $\mathrm{k}^{\text {th }}$ class adds significantly to model fit. For instance, the BLRT_p of 0.04 for the 4-class model indicates that adding a fourth class led to an improvement of model fit compared to the 3-class model that was just significant when using an alpha of 0.05. When using 'mclust' as a stand-alone package, the BLRT is not calculated by default, but can be obtained with the mclustBootstrapLRT function.

Next, we can run similar commands, with different values for the models argument. It is also possible to estimate more than one model variant in a single run by providing a vector of model numbers (e.g., models $=c(1,6))$.

The second approach to determine the model variant to be estimated is by using the variances and covariances arguments in the estimate_profiles command. The variances argument can have the values "equal" (i.e. variable variances constrained to be equal across classes) or "varying" (i.e. variances allowed to vary across classes). The covariances argument can have the values: "zero" (i.e. all variable covariances fixed to zero), "equal" (i.e. covariances constrained to be equal across classes) or "varying" (i.e. covariances allowed to vary across classes). Now, if we again want to estimate LPA models with 1 to 9 classes, with an EEI configuration, we can use:

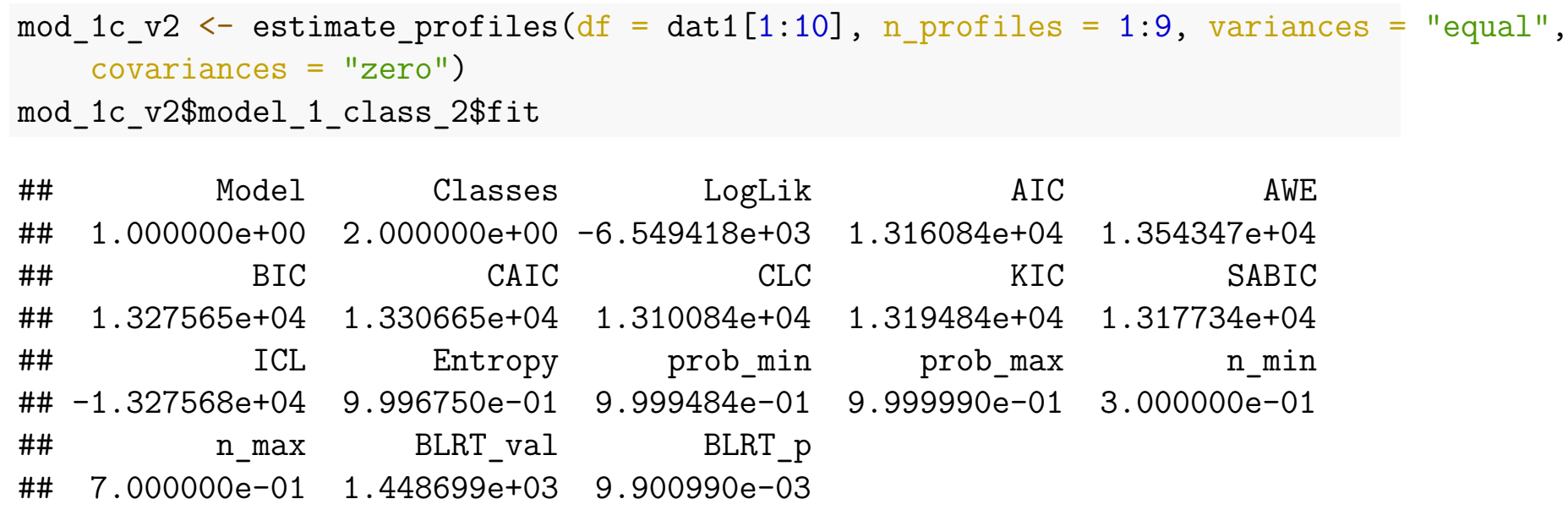

As expected, the output is exactly the same as the one we got when using the models argument. Now, if we want to, we can continue fitting other model variants using either approach. Next, we can manually compare the models in terms of their BIC and AIC values 
or we can use compare_solutions to do this in an automated fashion. Interestingly, the best model can be selected based on the integrated information about several fit indices (analytic hierarchy process; see help(tidyLPA : :AHP)) for more details). We obtain the model comparison with the following code.

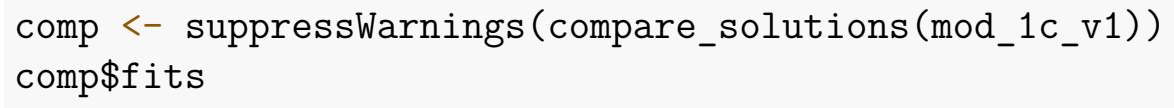

As shown in the 'mclust' examples above, the EEI model (models=1) with 3 classes fit the data best, not only according to the BIC, but according to the integrated information from a broader range of fit indices. Note that we did not fit any competing EEE, VVV and VVI models here. If we would have wanted to compare fit to these model variants too, we should have actively told the package to fit these too, either by specifying a vector of different types of models to fit in the models argument or by repeating the analysis with different values for the variances and covariances arguments.

\subsection{Comparison to Mplus}

For comparison, we fit the equivalent EEI model variants in Mplus. For this, we can mostly rely on the defaults of Mplus. We only need to specify that we want to estimate class-specific variable means. The variances are automatically constrained to be equal across classes and within class variable covariances are fixed to 0 . Here we have the separate codes for fitting the first 4 models wit 1-4 class models (code for the 5-9 class models not shown).

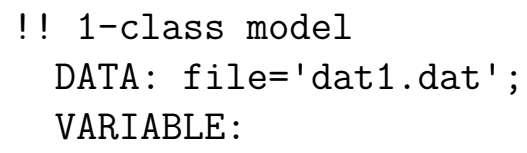




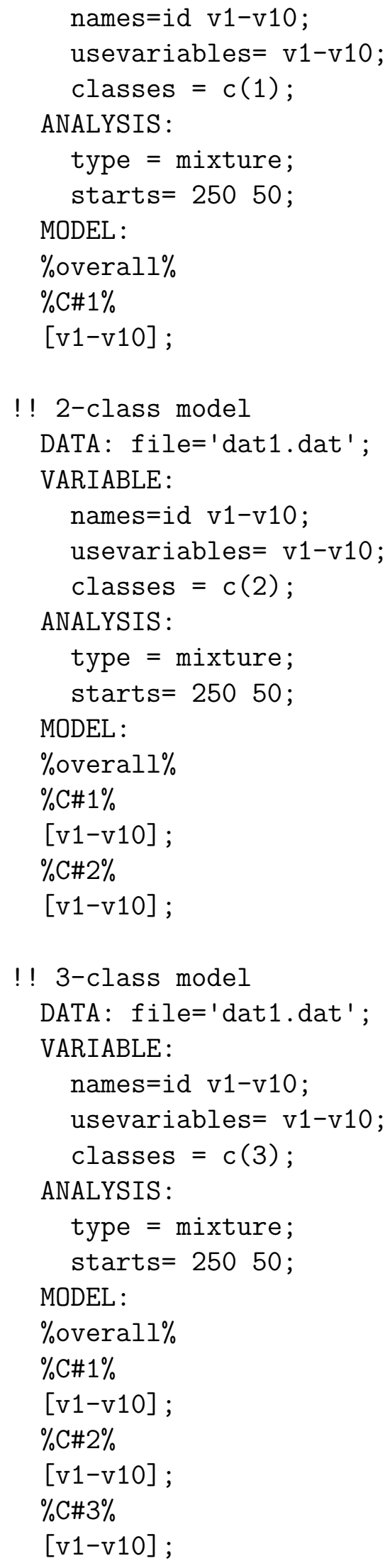

!! 3-class model

DATA: file='dat1.dat' ;

VARIABLE:

names $=i d \mathrm{v} 1-\mathrm{v} 10$;

usevariables $=\mathrm{v} 1-\mathrm{v} 10$;

classes $=c(3)$;

ANALYSIS :

type = mixture;

starts $=25050$;

MODEL :

$\%$ overall $\%$

$\%$ C\#1\%

[v1-v10];

$\% \mathrm{C} \# 2 \%$

[v1-v10];

$\% \mathrm{C} \# 3 \%$

[v1-v10]; 


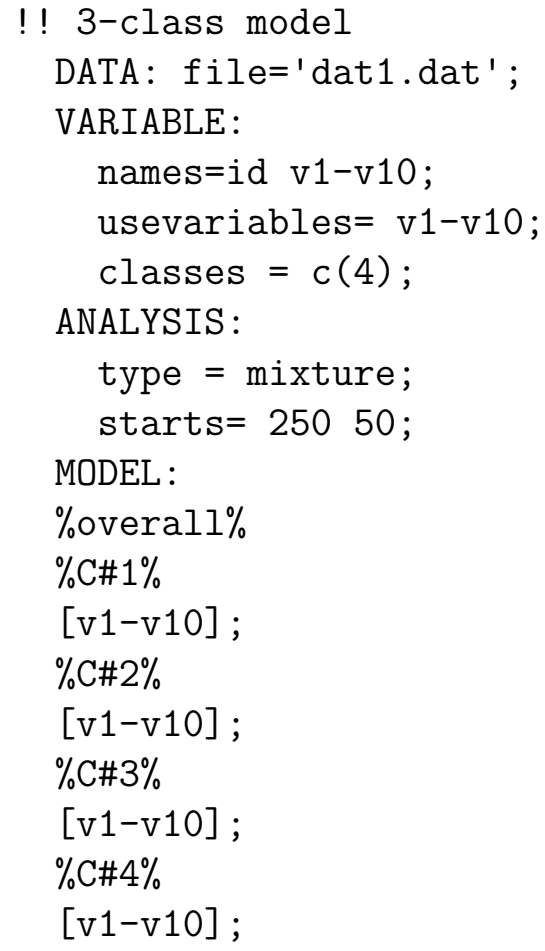

Each of the models was fitted with multiple initial stage random starts and multiple final stage optimizations to reduce the risk of finding solutions on a local maximum. For the 1-6 class models, 250 iniitial and 50 final starts were used. For the 7-class model 1000 initial starts and 200 final optimizations were used and for the 8-and 9-class models, a replicable global solution could only be obtained with 5000 initial stage starts and 1000 final stage optimizations. The fit indices for each of the fitted models is displayed in the table below. For now, we only look at the estimated fit indices.

\begin{tabular}{l|l|l}
\hline \multirow{2}{*}{ Classes } & \multicolumn{2}{|c}{ EEI models } \\
\cline { 2 - 3 } & AIC & BIC \\
\hline 1 & 14587.535 & 14587.535 \\
\hline 2 & 13160.836 & 13275.653 \\
\hline 3 & 11986.551 & 12142.11 \\
\hline 4 & 11957.71 & 12154.01 \\
\hline 5 & 11951.766 & 12188.808 \\
\hline 6 & 11944.632 & 12222.415 \\
\hline 7 & 11935.501 & 12254.027 \\
\hline 8 & 11929.428 & 12288.695 \\
\hline 9 & 11925.203 & 12325.212 \\
\hline
\end{tabular}

If we compare the BIC values for the EEI models to those obtained with ' $m$ clust' directly and via 'tidyLPA', we can see that the results are quite similar for the less complex models, with the BIC-values for the 2- and 3-class models being exactly the same in terms of their absolute values. When taking a closer look at the 3 -class model that was previously found to be the best model, we can also see that that the Mplus-based classifications $(n=100, n=110$, and $\mathrm{n}=90$ ) and entropy estimates (entropy $=1.0$ ) are similar to those obtained with ' mclust' 
and/or 'tidyLPA'.

If we look at the larger picture and evaluate the overlap between the BIC values obtained for all models with class numbers ranging from 1 to 9 , we can see that the estimated BIC values show more differences between the R-packages and Mplus for the models with larger numbers of classes. This can be explained by the different methods used by Mplus and 'mclust' /'tidyLPA' to generate the start values for estimation (see below). These differences can cause the packages to yield different results for more complex models and/or models that are increasingly misspecified (as the models with more than 3 classes were in our example).

\section{Final comments}

In this tutorial we have seen that it is relatively easy to run an LPA in R. As with all data-driven analyses, care should be taken not to over-interpret the results. In the end, LPA identifies classes in such a way as to optimally explain variance on a range of variables and not to optimize interpretability or usefulness.

Another remark with regard to LPA results is that class-membership is probabilistic in nature: each subject in an analyzed sample has a probability of being in each of the model's classes. Subjects can be allocated to a class based on their highest class-probability. Importantly, this can only be done with enough certainty if separation between classes is sufficient, which means that we see that each subject has a clear highest probability (e.g., $\mathrm{p}=0.9$ ) for one of the classes. The entropy statistic is often used to quantify this separation, with values $<0.8$ being taken to indicate insufficient separation to allocate subjects to one class. In such cases, the class probabilities themselves can still be used in further investigations.

Depending on one's preferences, one can choose either the 'mclust' or the 'tidyLPA' package. The latter may be especially attractive for persons that already have experience with Mplus or comparable software. It is important to note, though, that differences exist between the estimation approaches taken by both R-packages and by Mplus.

Mixture models such as LPA need to be estimated in an iterative fashion, using an EM algorithm that is very sensitive to the used start values, where poor start values can lead the estimation process to a poor solution. In addition, there is often a chance that the iterative EM process leads to a solution that is at a local, rather than a global maximum in the likelihood 'landscape'.

Different methods have been developed to initialize the estimation (get starting values) in such a way as to optimize the chance of arriving at an accurate model solution (Biernacki et al., 2003; Shireman et al., 2016). Mplus and 'mclust' take two different approaches. Mplus uses a 'brute force' approach and reruns the model with multiple sets of random start values, each generated from uniform distributions of values with ranges that are based on the data (Muthén \& Muthén, 1998-2015). The best solution is the model with the highest loglikelihood that was arrived upon from at least two different starting points. In contrast, ' $m c l u s t$ ' uses the data itself to generate a set of plausible start values using hierarchical clustering. This means that the start values are informed by the (hierarchical structure of) the data, which can work well. A downside is that only a single set of start values is used, so the risk of 
arriving at a local optimum is not addressed. These approaches show clear differences and have been shown previously to not always arrive at the same solutions, with some authors being especially critical of the 'mclust' approach (Shireman et al., 2017). In our example, we did not see much difference between the two approaches, but it should be noted that many real-world data sets do not have such a clear latent structure, making it much more challenging to arrive at an accurate solution, given the data.

Based on the provided examples and these technical differences between 'mclust' and Mplus, it is probably better to see 'mclust' and 'tidyLPA' as useful alternatives for Mplus, rather than as tools to exactly mimic what can be done with the latter software.

\section{References}

Banfield, J., Raftery, A.E. (1993). Model-based Gaussian and non-Gaussian clustering. Biometrics. 49: 803-821.

Biernacki,C.,Celeux,G., Govaert,G.(2003).Choosing starting values for the EM algorithm for getting the highest likelihood in multivariate Gaussian mixture models. Computational Studies and Data Analysis, 41, 561-575.

Celeux, G., Govaert, G. (1995) Gaussian parsimonious clustering models. Pattern Recognition. 28:781-793

Fraley, C., Raftery, A.E. (1998) How many clusters? Which clustering method? Answers via model-based cluster analysis. The Computer Journal 41: 578-588.

Gibson, W. A. (1959). Three multivariate models: Factor analysis, latent structure analysis, and latent profile analysis. Psychometrika, 24, 229-252.

Linzer, D.A., Lewis J. (2011). "poLCA: an R Package for Polytomous Variable Latent Class Analysis." Journal of Statistical Software. 42(10): 1-29

Meyer, D., Dimitriadou, E., Hornik, K., Weingessel, A., Leisch, F. (2019). e1071: Misc Functions of the Department of Statistics, Probability Theory Group (Formerly: E1071), TU Wien. R package version 1.7-3. https://CRAN.R-project.org/package=e1071

Muthén, L., Muthén, B., 1998-2015. Mplus User's Guide, 7th ed., Muthén \& Muthén, Los Angeles.

Neale, M.C., Hunter, M.D., Pritikin, J.N., Zahery, M., Brick, T.R., Kirkpatrick, R.M., Estabrook, R., Bates, T.C., Maes, H.H., Boker, S.M. (2016). "OpenMx 2.0: Extended structural equation and statistical modeling." Psychometrika 81(2): 535-549.

Nylund-Gibson, K., Choi, A. Y. (2018). Ten frequently asked questions about latent class analysis. Translational Issues in Psychological Sciences, 4, 440-461.

Oberski, D. (2016). Mixture models: Latent profile and latent class analysis. In J. Robertson \& M. Kaptein (Eds.), Modern statistical methods for HCI (pp. 275-287). Cham, Switzerland: Springer Inter-national Publishing. 
Pastor, D.A., Barron, K.E., Miller, B.J., Davis, S.L. (2007). A latent profile analysis of college students' achievement goal orientation. Contemporary Educational Psychology 32(1): $8-47$.

Rosenberg, J.M., Beymer, P.N., Anderson, D.J., Van Lissa, C.J., \& Schmidt, J.A. (2018). tidyLPA: An R Package to Easily Carry Out Latent Profile Analysis (LPA) Using Open-Source or Commercial Software. Journal of Open Source Software 3(30): 978.

Scrucca, L., Fop M., Murphy, T.B., Raftery A.E. (2016) mclust 5: clustering, classification and density estimation using Gaussian finite mixture models The R Journal 8/1: 289-317.

Shireman, E. M., Steinley, D., \& Brusco, M. J. (2016). Local Optima in Mixture Modeling. Multivariate Behavioral Research 51(4): 466-481.

Shireman, E., Steinley, D., Brusco, M.J. (2017). Examining the effect of initialization strategies on the performance of Gaussian mixture modeling. Behavioral Research Methods 49(1):282-293.

Sterba, S.K. (2013). Understanding Linkages Among Mixture Models. Multivariate Behav Res. 2013 48(6): 775-815.

Wolfe, J. (1970). Pattern clustering by multivariate mixture analysis. Multivariate Behavioral Research, 5: 329-350.

\section{Appendix}

$\mathrm{R}$ code to simulate the tutorial data. Make sure to install the 'MASS' and 'reshape2' packages first.

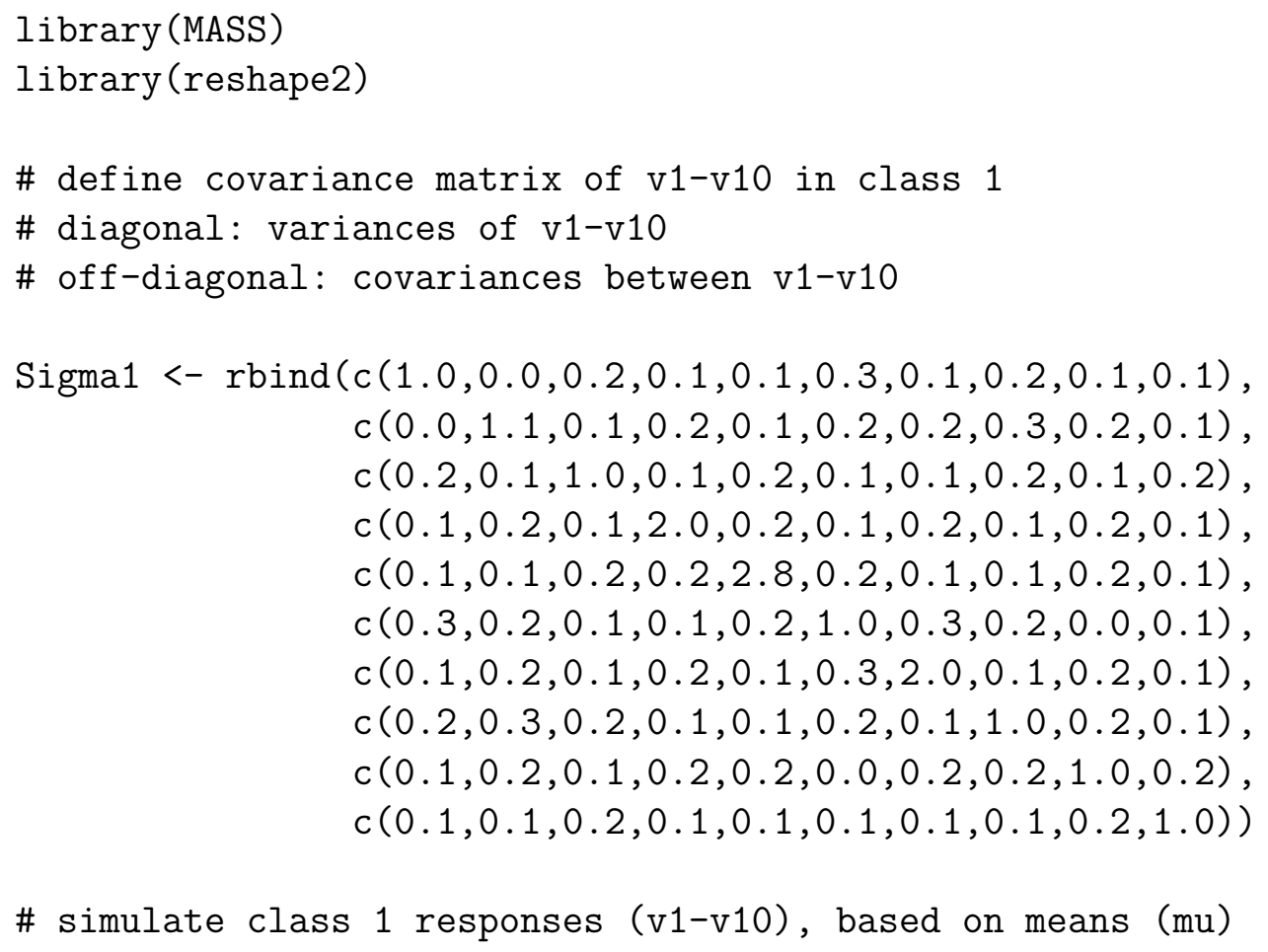


\# and covariance matrix (sigma)

set.seed (0111)

cl1 <-mvrnorm(100, mu=c $(2,3,2,6,7,4,5,8,2,1)$, Sigma=Sigma1)

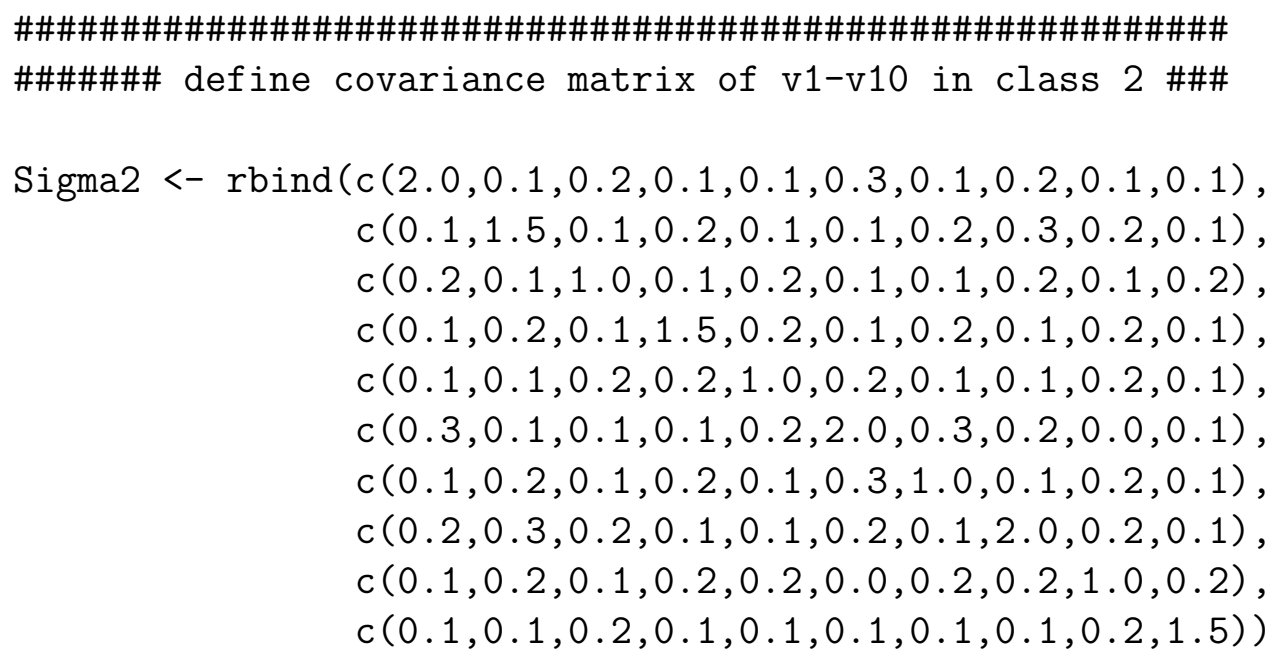

\# simulate class 2 responses (v1-v10), based on means (mu) \# and covariance matrix (sigma)

set.seed (0111)

c12<-mvrnorm(110, mu=c $(6,7,1,3,2,8,3,8,2,5)$, Sigma=Sigma2)

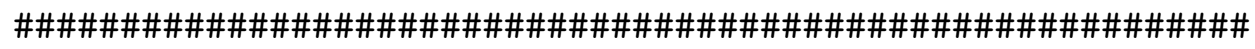

\#\#\#\#\#\#\# define covariance matrix of v1-v10 in class 3 \#\#\#

Sigma3 <- $\operatorname{rbind}(c(1.0,0.1,0.2,0.1,0.1,0.3,0.1,0.3,0.1,0.1)$, $c(0.1,1.0,0.1,0.2,0.1,0.1,0.2,0.3,0.2,0.1)$, $c(0.2,0.1,2.2,0.1,0.2,0.1,0.1,0.2,0.1,0.2)$, $c(0.1,0.2,0.1,2.0,0.1,0.1,0.2,0.1,0.2,0.1)$, $c(0.1,0.1,0.2,0.1,1.0,0.2,0.1,0.1,0.2,0.1)$, $c(0.3,0.1,0.1,0.1,0.2,1.0,0.3,0.2,0.0,0.1)$, c $(0.1,0.2,0.1,0.2,0.1,0.3,1.0,0.1,0.2,0.1)$, c $(0.3,0.3,0.2,0.1,0.1,0.2,0.1,1.2,0.2,0.1)$, $c(0.1,0.2,0.1,0.2,0.2,0.0,0.2,0.2,3.0,0.2)$, $c(0.1,0.1,0.2,0.1,0.1,0.1,0.1,0.1,0.2,2.0))$

\# simulate class 3 responses (v1-v10), based on means (mu) \# and covariance matrix (sigma)

set.seed (0111)

cl3 <-mvrnorm(90, mu=c $(2,5,8,7,5,2,1,4,9,8)$, Sigma=Sigma3)

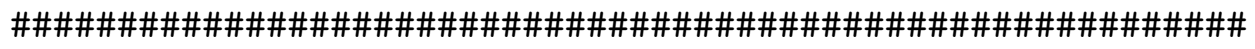

\#\#\# convert matrices to data.frame objects \& add a class $\mathrm{nr}$ cl1 <- as.data.frame (cl1) 


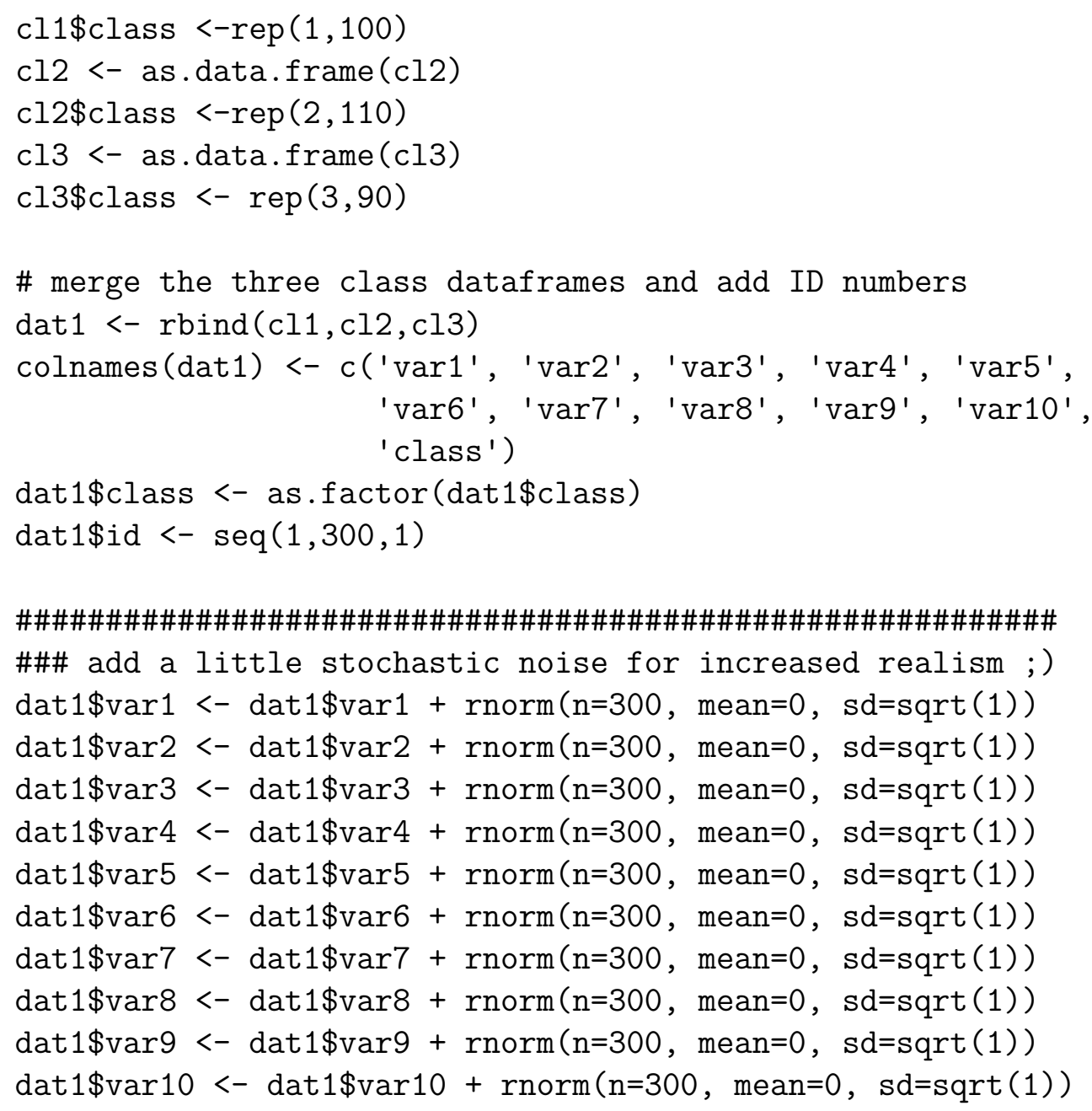

